\title{
Multiple Sklerose und Salz
}

Fragestellung: Spielt Salz eine besondere Rolle bei Autoimmunerkrankungen, insbesondere bei der Multiplen Sklerose (MS)?

Hintergrund: Auf einen möglichen Zusammenhang von verstärkter Autoimmunität und hohem Salzgehalt wurde schon mehrfach hingewiesen. Initial konnte gezeigt werden, dass TLymphozyten bei hoher Salzkonzentration wesentlich stärker proliferierten als im salzfreien oder -reduzierten Milieu. Davon schienen insbesondere TH17-Zellen und Makrophagen betroffen. Auch die Tatsache, dass in den letzten Jahrzehnten zunehmend Autoimmunerkrankungen in den westlichen, neuerdings aber auch in Dritte-Welt-Ländern zu beobachten sind, könnte durch den Lebensstil mit salzhaltiger Kost - zumindest partiell - erklärt werden. Mehrere Studien widmeten sich in den letzten Monaten diesem Thema.

1 Hucke $S$ et al. Sodium chloride promotes pro-inflammatory macrophage polarization thereby aggravating CNS autoimmunity. J Autoimmun 2016; 67: 90 - 101

2 Binger KJ et al. High salt reduces the activation of IL-4- and IL13-stimulated macrophages. J Clin Invest 2015; 125: 4223-38

3 Hernandez AL et al. Sodium chloride inhibits the suppressive function of FOXP3+ regulatory T cells. J Clin Invest. 2015; 125: $4212-22$

4 Farez MF et al. Sodium intake is associated with increased disease activity in multiple sclerosis. J Neurol Neurosurg Psychiatry 2015; 86: 26-31
Ergebnisse: In einer aktuellen Studie [1] wurde das Verhalten von Makrophagen in Abhängigkeit von der Salzkonzentration im klassischen EAE-Modell untersucht. Im Tierexperiment war eine salzreiche Diät mit einer stärkeren Aktivierung von Makrophagen, aber auch mit einer deutlich verstärkten Infiltration von Makrophagen in das ZNS assoziiert, was jeweils auch mit einer verstärkten Erkrankungsprogredienz verbunden war. Innerhalb der Makrophagen bewirkte das $\mathrm{NaCl}$-reiche Milieu eine ver- stärkte proinflammatorische und erhöhte Zytokinproduktion. Zudem konnte eine erhöhte Freisetzung immunstimulierender Moleküle und antigenunabhängige Verstärkung der T-Zell Proliferation beobachtet werden. Die salzinduzierten proinflammatorischen Makrophagen zeigten ferner eine erhöhte Aktivierung von NF-kB und der MAPK-Signalwege. Die Gabe dieser Zellen in ein anderes Tier mit frisch induzierter EAE führte zu einem deutlich schwereren Krankheitsverlauf als die Gabe „unkonditionierter" Makrophagen. Auch bei humanen Monozyten führte ein hoher NaCl-Gehalt zu einer proinflammatorischen Reaktion und verstärkten Induktion von T-Zell-Proliferation.

Eine andere Arbeitsgruppe [2] fand heraus, dass hohe $\mathrm{NaCl}$ Konzentrationen die Aktivierung von IL-4- und IL-13-stimulierten Makrophagen reduzieren und damit die antiinflammatorischen M2-Makrophagen inhibieren. Auch FOXP3 ${ }^{+}$-TregsZellen, die für das Gleichgewicht von Selbsttoleranz und Autoimmunität wichtig sind, sind bei hohen $\mathrm{NaCl}$-Konzentrationen offensichtlich gestört [3]. Im Tiermodell stieg die Sekretion des inflammatorischen Zytokins IFN $\gamma$ an. Damit scheinen hohe $\mathrm{NaCl}$-Konzentrationen nicht nur direkt proinflammatorische Mechanismen zu induzieren oder zu verstärken, sondern auch antiinflammatorische Mechanismen zu inhibieren und die Funktion T-regulatorischer Zellen zu stören.

Eine weitere Studie [4] untersuchte die Salzausscheidung im Urin von 70 MS-Patienten als Korrelat der regelmäßigen quantitativen Salzzufuhr über den Zeitraum von zwei Jahren. Bei hoher oder mittlerer Ausscheidung war die Schubrate 2,75fach höher und das Risiko, eine neue T2-Läsion zu entwickeln, 3,4fach höher als bei niedriger Ausscheidung. Die Untersuchung einer anderen Kohorte mit 52 MS-Patienten ergab die gleichen Resultate.

Schlussfolgerungen: Salz scheint autoimmunologische Prozesse zu beeinflussen. Die erste klinische Studie am Menschen scheint die tierexperimentellen Daten zu bestätigen.

\section{- Kommentar von Volker Limmroth, Köln}

\section{Salzreduzierte Kost für Patienten mit Autoimmunerkrankungen?}

Die Daten beim Menschen sind noch sehr begrenzt, aber man kommt nicht umhin festzustellen, dass das gesamte Datenmaterial zum Thema Salz und Autoimmunität recht homogen und nicht widersprüchlich ist. Bei den In-vitro-Experimenten und Tierversuchen mag man noch einwenden, dass es sich hier teilweise um Dosierungen handelt, die kein halbwegs bewusstseinsklarer Mensch freiwillig einnehmen würde. Die Daten aus der Urinanalyse stimmen aber nachdenklich, auch wenn sie von anderen Gruppen sicherlich noch bestätigt werden müssen.

Es ist ein bisschen wie beim Vitamin D: Eigentlich klingt es zu banal, als dass es wahr sein könnte, aber nun sind tatsächlich dringend große klinische Studien notwendig, die diese Hypothese klinisch prospektiv überprüfen. Sollte durch die „einfache“ Reduktion von Salz eine autoimmunologische Aktivität spürbar reduzierbar sein, müsste diese Verhaltensänderung unseren Patienten dringend empfohlen werden.

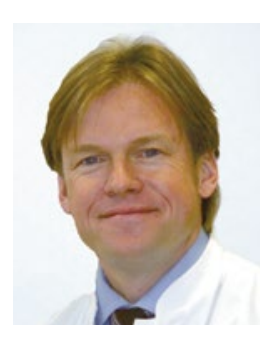

Prof. Dr. med. Volker Limmroth, Köln-Merheim

Chefarzt der Klinik für Neurologie und Palliativmedizin Köln-Merheim E-Mail: LimmrothV@kliniken-koeln.de 